\title{
Multilevel Image Thresholding for Image Segmentation by Optimizing Fuzzy Entropy using Firefly Algorithm
}

\author{
M.S.R. Naidu ${ }^{1 *}$, P. Rajesh Kumar ${ }^{2}$ \\ Department of Electronics and communication Engineering \\ ${ }^{1}$ Aditya institute of technology and management (AITAM), Tekkali, Andhrapredesh, India \\ ${ }^{2}$ A.U.College of engineering (A), Andhra university, Visakhaptnam, Andhrapredesh, India \\ E-mails:msrnaidu312@rediffmail.com¹, rajeshauce@gmail.com²
}

\begin{abstract}
Image thresholding is the process of extracting objects in a scene from the background accompanies for the analysis and interpretation of image which is mostly employed for its advanced simplicity, robustness, less convergence time and accuracy. The main intend of image segmentation is to segregate the foreground from background. As ordinary thresholding method of image segmentation is computationally expensive while extending for multilevel image thresholding, the need for optimization techniques is highly recommended. The so called optimization techniques such as Particle swarm optimization and bat algorithm undergo instability when the particle velocity is maximum and stagnation stage attributable to quick exploration. This paper proposes for the first time the multilevel image thresholding for image segmentation by using Fuzzy entropy maximized by naturally inspired firefly algorithm. A firefly based multilevel image thresholding is established by maximizing Fuzzy entropy where the results are proved better in misclassification, standard deviation, Structural Similarity Index and segmented image quality while comparing with differential evolution, Particle swarm optimization and bat algorithm..
\end{abstract}

Keywords: Image thresholding; Image segmentation; Fuzzy entropy; Bat algorithm; Firefly algorithm

\section{INTRODUCTION}

Image thresholding is the process of extracting objects in a scene from the background that helps for analysis and interpretation of image. It is a challenging task for the researchers in image processing to select a preeminent gray level threshold that extracts the object from the background of the gray level image or color image. Selection of threshold is moderately simple in the case where histogram of the image has a deep valley representing background and sharp edges representing objects, but due to the multimodality of the histograms of many image selections of a threshold is difficult task. So researchers proposed many techniques for preeminent gray level threshold. Though there are so many segmentation techniques are in the literature, thresholding is mostly used for its advanced simplicity, robustness, less convergencetime and accuracy. Thresholding approaches are of two types that are nonparametric and parametric. In nonparametric approach thresholding is performed based on class variance as in otsu's technique or based on an entropy criterion, such as Shannon entropy, Fuzzy entropy and Kapur's entropy (Luca and Termini, 1972). If the image is partitioned into two classes, i.e. object and background, then the threshold is called bi-level threshold else multi-level threshold. Thresholding technique has so many real time applications like data, image and video compression, image recognition, pattern recognition, and image understanding and communication (chiranjeevi and jena, 2016). Sezgin and Sankur, (2004) performed comparative study on image thresholding and classified the image thresholding into six categories such as Histogram shape-based methods, Clustering-based methods, Entropybased methods, object attribute-based methods, spatial methods and local methods. Kapur classifies the image into some classes by calculating threshold which is based on the histogram of the gray level image (Kapur, 1985). Otsu's method classifies the image into some classes by calculating threshold which is based on betweenclass variance of the pixel intensities of that class (Otsu, 1979). These two methods are under the category of bilevel thresholding and found efficient in case of two thresholds, but for multi-level thresholding, the computational complexity is very high. Entropy may be a Shnnon, fuzzy, between class variations, Kapur's entropy, minimization of the Bayesian error and Birge-Massartthresholding strategy. The disadvantage of these techniques is that convergence time or computational time or CPU time is exponentially increasing with the problem. So alternative to these techniques which minimizes the CPU time for the same problem is evolutionary and swarm-based calculation techniques.

Sathya and Kayalvizhi (2011) applied bacterial foraging optimization algorithm (BF) for optimizing objective functions (Kapur's and Otsu's entropy), so achieved an efficient image segmentation. Further to improve convergence speed and the global searching ability of BF, they modified swarming step and reproduction step, thereby improved the robustness of BF and achieved fast convergence. Sathya and Kayalvizhi (2011) proposed Magnetic Resonance (MR) brain image segmentation by optimizing the multilevel thresholding 
using amended bacterial foraging (ABF) algorithm. The optimal thresholds are obtained by maximizing the Kapur's or Otsu's entropy with the help of ABF algorithm. The results are compared and proved better in the separation of gray, white and cerebrospinal fluid in MRI image for recognition as well as to diagnosis the disease. The same authors employed some modifications to bacterial foraging (BF) for Segmentation of brain magnetic resonance images (Sathya and Kayalvizhi, 2011). They did adaptive variation of step size of bacteria instead of fixed step size which is followed by ordinary bacterial foraging (Sathya and Kayalvizhi, 2011). Mbuyamba et al. (2016) used Cuckoo Search (CS) algorithm for energy minimization of alternative Active Contour Model (ACM) for global minimum and exhibited that polar coordinates with CS is better than rectangular. Among many optimization techniques are available in the literature, a few are used for bi-level thresholding for ordinary image segmentation, Ye et al. (2015) used fuzzy entropy with bat algorithm (BA) and compared the results with Artificial Bee Colony Algorithm (ABC), Ant Colony (ACO), Particle Swarm Optimization (PSO) and Genetic Algorithm (GA). Agrawal et al. (2013) used Tsallis entropy with CS algorithm and compared the results with BF, PSO and GA. Horng, (2010) used Firefly Algorithm (FA) for multilevel image thresholding. Kapur's and Otsu's entropy methods are simple and effective but computationally expensive when extended to multilevel thresholding because they employed an exhaustive search for optimal thresholds. So Hussein et al. (2016) developed a new strategy which reduces computational time of Kapur's and Otsu's entropy with the help of modified Bees Algorithm (MBA) called the Patch-Levy-based Bees Algorithm (PLBA) and the results showed much faster compared to ordinary Bees Algorithm. Whereas Ashish et al. (2014) used Kapur's entropy with CS and wind driven optimization (WDO) for multilevel thresholding of satellite image segmentation. Bhandari et al. (2015) proposed a Tsallis entropy based multilevel thresholding for colored satellite image segmentation using high dimensional problem optimizer that is Differential Evolution (DE), WDO, PSO and Artifical Bee Colony (ABC). The same authors carried out gray scale satellite image segmentation using modified artificial bee colony (MABC) by optimizing the Kapur's, Otsu and Tsallis entropy and compared the result with ABC, PSO and GA (Bhandari et al. 2015). The drawback of DE is constant tuning parameters (scaling factor (F) and crossover rate (CR)). So Ayala et al. (2015) vary the parameters of DE that follow beta probability distribution function. The beta distribution is flexible for modeling data that are measured in a continuous scale on a truncated interval in range $[0,1]$. They compared the beta differential evolution (BDE) based segmentation with fractional-order Darwinian particle swarm optimization (PSO). Li et al. (2015) proposed a modification to PSO that is dynamic-context cooperative quantum-behaved particle swarm in which updating of context vector is dynamic for Otsu's entropy based medical image segmentation. Sun et al. (2016) hybridize the gravitational search algorithm (GS) with genetic algorithm for multi-level thresholding of ordinary images for effective segmentation. They took the advantage and disadvantage of both the algorithms and proper hybridization which has resulted into the best segmentation compared to other methods. Akay (2013) proposed PSO and ABC based image segmentation using Kapur's and Otsu's entropy but PSO performance is further improved with position-velocity model which is based on inherent communication mechanism of celllike P systems of PSO (Penga et al., 2015). Ouadfel and Ahmed (2016) used social spiders optimization and flower pollination algorithm for multilevel image thresholding by optimizing Kapur's and Otsu's entropy and compared the results with BA and PSO. Saha et al. (2014) proposed Quantum Inspired Genetic Algorithm, Particle Swarm Optimization, Differential Evolution, Ant Colony Optimization, Simulated Annealing and Tabu Search with Otsu method, maximum tsallis entropy thresholding and proved that Quantum Inspired Particle Swarm Optimization is better than others by statistical test and Friedman test measures which reduce the computational complexities.

For the first time in this paper the researchers have applied Firefly algorithm (FA) for image thresholding by optimizing the Fuzzy entropy and compared the results with previous optimization techniques such as DE, PSO and BA. For the performance evolution of proposed firefly algorithm based image thresholding, we considered objective function value, standard deviation, structural similarity index, peak signal to noise ratio, misclassification error and computational complexity. In all performance measuring parameters the proposed algorithm performance is better when compared to other DE, PSO and BA.

\section{PROBLEM FORMULATION OF OPTIMUM THRESHOLDING METHODS}

Image thresholding is a process of converting a grayscale input image to a black and white image by using optimal thresholds. Thresholding may be a local or global but these methods are computationally expensive, so there is a need of optimization techniques which optimize the objective function results in the reduction of computational time of local or global methods. The optimization techniques find the optimal thresholds by maximizing the objective function such that segmented image clearly distinguishes the background and foreground of image. In this paper, researchers have chosen Fuzzy entropy as objective functions on which optimization techniques works. Let us assume an image that contains $\mathrm{L}$ gray levels and the range of these gray levels are $\{0,1,2, \ldots,(L-1)\}$. Then probability $P_{i}=h(i) / N(0<i<(L-1))$, where $h(i)$ denotes number of pixels for the corresponding gray-level $L$ and $N$ denotes total number of pixels in the image which is equal to $\sum_{i=0}^{L-1} h(i)$ 


\section{A. Concept of Fuzzy Entropy}

Let $D=\{(i, j): i=0,1,2, \ldots \ldots, M-1 ; j=0,1,2, \ldots \ldots . N-1\}$ and $G=\{0,1,2, \ldots \ldots, L-1\}$, Where $M$ is width of image, $\mathrm{N}$ is height of image and $L$ is number of gray level in image. $I(x, y)$ is the intensity of image at position $(x, y)$ and $D_{k}$ $=\{(x, y): I(x, y)=k,(x, y)=D\}, k=0,1,2, \ldots \ldots, L-1$. Let us assume two thresholds i.e. $\mathrm{T}_{1}, \mathrm{~T}_{2}$ which divide the domain $D$ of the original image into three regions such as $E_{d}, E_{m}$ and $E_{b}$. $E_{d}$ region covers the pixels whose intensity value is less than $T_{1}, E_{m}$ contains the pixels whose intensity is in between $T_{1}, T_{2}$ and $E_{b}$ covers the pixels whose intensity is greater than $\mathrm{T}_{2} . \Pi_{3}=\left\{\mathrm{E}_{\mathrm{d}}, \mathrm{E}_{\mathrm{m}}, \mathrm{E}_{\mathrm{b}}\right\}$ is an unknown probabilistic partition of $\mathrm{D}$ whose probability distribution is given as (Zhao et. al, 2001) $P_{d}=P\left(E_{d}\right) P_{m}=P\left(E_{m}\right) P_{b}=P\left(E_{b}\right) . \mu_{\mathrm{d}}, \mu_{\mathrm{m}}$ and $\mu_{\mathrm{b}}$ are the membership functions $(\mu)$ of $E_{d}, E_{m}$ and $E_{b}$ respectively and require six parameters like $a_{1}, b_{1}, c_{1}, a_{2}, b_{2}, c_{2}$. The thresholds $T_{1}$ and $T_{2}$ values are variable based on the membership functions. For each $k=1,2, \ldots ., 255$, let

$$
\begin{aligned}
& D_{d}=\left\{(x, y): I(x, y) \leq T_{1},(x, y) \in D_{k}\right\} \\
& D m=\left\{(x, y): T_{I}<I(x, y) \leq T 2,(x, y) \in D k\right\} \\
& D b=\{(x, y): I(x, y)>t 2,(x, y) \in D k\}
\end{aligned}
$$

If the conditional probability of $E_{d}, E_{m}$ and $E_{b} i s p_{d k}, p_{m \mid k}$ and $p_{b \mid k}$ respectively under the circumstance that the pixel pertains to $D_{k}$ with $p_{d \mid k}+p_{m \mid k}+p_{b \mid k}=1(k=0,1,2, \ldots \ldots, 255)$ then above equations can be rewritten as

$$
\begin{aligned}
& p_{k d}=p\left(D_{p}\right)=p_{k} \times p_{d / k} \\
& p_{k m}=p\left(D_{m}\right)=p_{k} \times p_{m / k} \\
& p_{k b}=p\left(D_{b}\right)=p_{k} \times p_{b / k}
\end{aligned}
$$

Let the grade of pixels with gray level value of $k$ belong to the class dark $\left(E_{d}\right)$, dust $\left(E_{m}\right)$ and bright $\left(E_{b}\right)$ be equivalent to their conditional probability $\mathrm{p}_{\mathrm{d} \mid \mathrm{k}}, \mathrm{p}_{\mathrm{m} \mid \mathrm{k}}$ and $\mathrm{p}_{\mathrm{b} \mid \mathrm{k}}$ respectively. Then the following equations will hold as:

$$
\begin{aligned}
& p_{d}=\sum_{k=0}^{255} p_{k} * p_{d / k}=\sum_{k=0}^{255} p_{k} * \mu_{d}(k) \\
& p_{m}=\sum_{k=0}^{255} p_{k} * p_{m / k}=\sum_{k=0}^{255} p_{k} * \mu_{m}(k) \\
& p_{b}=\sum_{k=0}^{255} p_{k} * p_{b / k}=\sum_{k=0}^{255} p_{k} * \mu_{b}(k)
\end{aligned}
$$

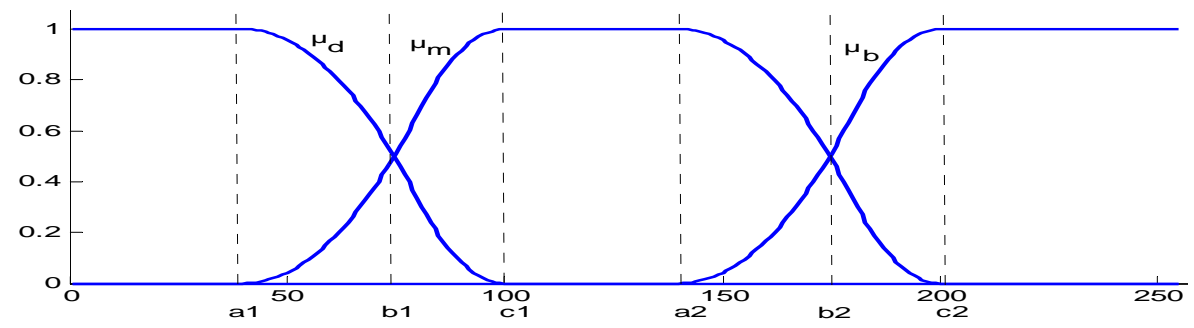

Fig. 1.Membership function graph with $\mathrm{a} 1=40 ; \mathrm{b} 1=80 ; \mathrm{c} 1=100 ; \mathrm{a} 2=140 ; \mathrm{b} 2=180 ; \mathrm{c} 2=200$

The fuzzy membership functions is drawn and shown in Fig. 1. The function $Z\left(k, a_{1}, b_{1}, c_{1}, a_{2}, b_{2}, c_{2}\right), U\left(k, a_{1}\right.$, $\left.\mathrm{b}_{1}, \mathrm{c}_{1}, \mathrm{a}_{2}, \mathrm{~b}_{2}, \mathrm{c}_{2}\right)$ and $\mathrm{S}\left(\mathrm{k}, \mathrm{a}_{1}, \mathrm{~b}_{1}, \mathrm{c}_{1}, \mathrm{a}_{2}, \mathrm{~b}_{2}, \mathrm{c}_{2}\right)$ are assigned as membership functions of class dark $\mu_{\mathrm{d}}(\mathrm{k})$, dust $\mu$ $\mathrm{m}(\mathrm{k})$ and bright $\mu_{\mathrm{b}}(\mathrm{k})$ respectively. Then the membership functions is given as

$$
\mu_{d}(\mathrm{k})=\left\{\begin{array}{cc}
1 & k \leq a_{1} \\
1-\frac{\left(k-a_{1}\right)^{2}}{\left(\mathrm{c}_{1}-\mathrm{a}_{1}\right) *\left(\mathrm{~b}_{1}-\mathrm{a}_{1}\right)} & a_{1}<k \leq b_{1} \\
\frac{\left(k-c_{1}\right)^{2}}{\left(\mathrm{c}_{1}-\mathrm{a}_{1}\right) *\left(\mathrm{c}_{1}-b_{1}\right)} & b_{1}<k \leq c_{1} \\
0 & k>c_{1}
\end{array}\right.
$$




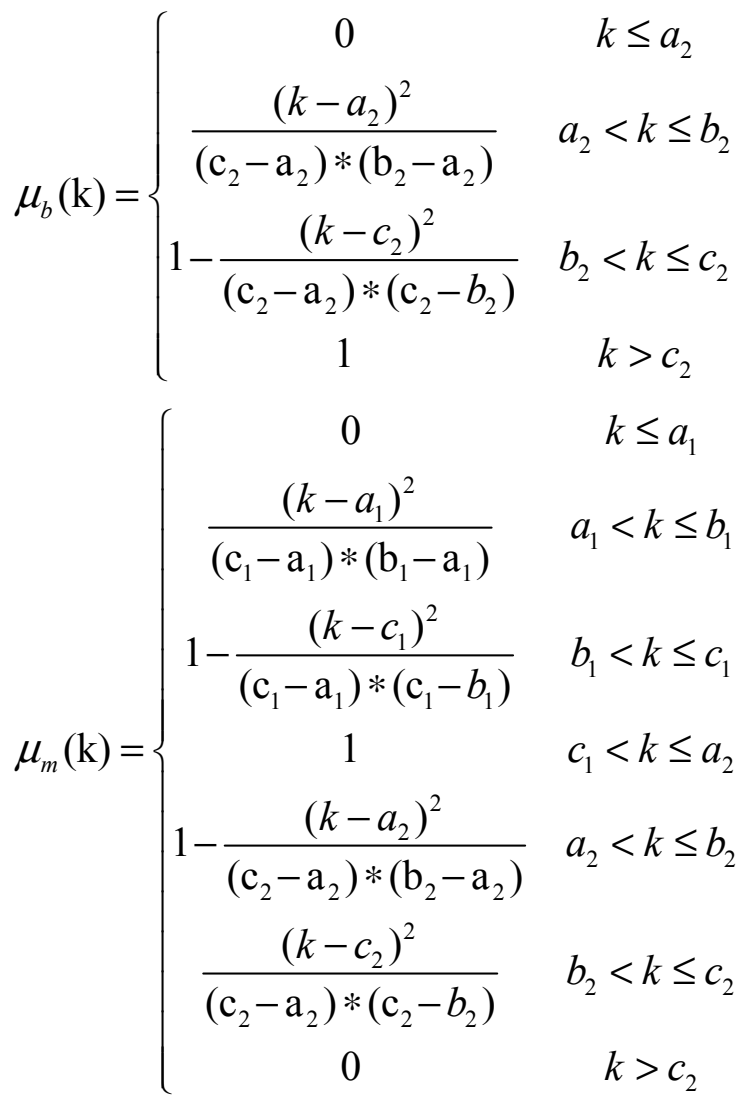

The above said equations are written by assuming $0 \leq \mathrm{a}_{1}<\mathrm{b}_{1}<\mathrm{c}_{1}<\mathrm{a}_{2}<\mathrm{b}_{2}<\mathrm{c}_{2} \leq 255$. Then, the fuzzy entropy function of each class could be given as (Tao et al., 2007)

$$
\begin{gathered}
H_{d}=-\sum_{k=0}^{255} \frac{p_{k^{*} \mu_{d}}(k)}{p_{d}} * \ln \left(\frac{p_{k^{*} \mu_{d}(k)}}{p_{d}}\right) \\
H_{m}=-\sum_{k=0}^{255} \frac{p_{k^{*} \mu_{m}(k)}}{p_{m}} * \ln \left(\frac{p_{k^{*} \mu_{m}(k)}}{p_{m}}\right) \\
H_{b}=-\sum_{k=0}^{255} \frac{p_{k^{*} \mu_{b}(k)}}{p_{b}} * \ln \left(\frac{p_{k^{*} \mu_{b}(k)}}{p_{b}}\right)
\end{gathered}
$$

The whole fuzzy entropy is calculated through summarizing fuzzy entropy of each class i.e.

$$
\mathrm{H}\left(\mathrm{a}_{1}, \mathrm{~b}_{1}, \mathrm{c}_{1}, \mathrm{a}_{2}, \mathrm{~b}_{2}, \mathrm{c}_{2}\right)=\mathrm{H}_{\mathrm{d}}+\mathrm{H}_{\mathrm{m}}+\mathrm{H}_{\mathrm{b}}
$$

The above equation is an objective function which is to be optimized with the optimization techniques. Optimization techniques optimize or maximize $\mathrm{H}\left(\mathrm{a}_{1}, \mathrm{~b}_{1}, \mathrm{c}_{1}, \mathrm{a}_{2}, \mathrm{~b}_{2}, \mathrm{c}_{2}\right)$ function by varying $\mathrm{a}_{1}, \mathrm{~b}_{1}, \mathrm{c}_{1}, \mathrm{a}_{2}, \mathrm{~b}_{2}, \mathrm{c}_{2}$. Once these values are optimized, then threshold values are calculated with the following equation

$$
\mu_{\mathrm{d}}\left(\mathrm{T}_{1}\right)=\mu_{\mathrm{m}}\left(\mathrm{T}_{1}\right)=0.5 \text { and } \mu_{\mathrm{m}}\left(\mathrm{T}_{2}\right)=\mu_{\mathrm{b}}\left(\mathrm{T}_{2}\right)=0.5
$$

From Fig. 1 it is observed that $T_{1}$ and $T_{2}$ are the point of interaction of $\mu_{d}(k), \mu_{m}(k)$ and $\mu_{b}(k)$ curve. From Eqs (10)-(12), the values of $\mathrm{T}_{1}$ and $\mathrm{T}_{2}$ calculated with the below equation

$$
\begin{aligned}
& T_{1}= \begin{cases}a_{1}+\sqrt{\left(\mathrm{c}_{1}-\mathrm{a}_{1}\right) *\left(\mathrm{~b}_{1}-a_{1}\right) / 2} & \left.\left(\mathrm{a}_{1}+\mathrm{c}_{1}\right) / 2 \leq b_{1} \leq c_{1}\right) \\
c_{1}-\sqrt{\left(\mathrm{c}_{1}-\mathrm{a}_{1}\right) *\left(\mathrm{c}_{1}-b_{1}\right) / 2} & \mathrm{a}_{1} \leq b_{1} \leq\left(\mathrm{a}_{1}+\mathrm{c}_{2}\right) / 2\end{cases} \\
& T_{2}= \begin{cases}a_{2}+\sqrt{\left(\mathrm{c}_{2}-\mathrm{a}_{2}\right) *\left(\mathrm{~b}_{2}-a_{2}\right) / 2} & \left(\mathrm{a}_{2}+\mathrm{c}_{2}\right) / 2 \leq b_{2} \leq c_{2} \\
c_{2}-\sqrt{\left(\mathrm{c}_{2}-\mathrm{a}_{2}\right) *\left(\mathrm{c}_{2}-b_{2}\right) / 2} & \mathrm{a}_{2} \leq b_{2} \leq\left(\mathrm{a}_{2}+\mathrm{c}_{2}\right) / 2\end{cases}
\end{aligned}
$$

As per the requirements of researchers, the two level thresholding can be extended to three or more and can be restricted to single level also. For two thresholds the number of parameters to be optimized is six and as levels of increasing number parameters to be optimized is also increasing, so fuzzy entropy takes much time for convergence. Hence two level image thresholding for image segmentation with the Fuzzy entropy proved to be 
efficient and effective but for multilevel thresholding, entropy technique consume much convergence time and increase exponential with level of thresholds. The drawback of Fuzzy entropy is convergence time. To improve the performance of these methods further and to reduce the convergence time, researchers used applications of optimization techniques such as differential evolution, Particle swarm optimization, Bat algorithm and Firefly algorithm for image thresholding and henceforth image segmentation. This techniqueis set to maximize the Fuzzy entropy as given in (16).

\section{OVERVIEW OF FIREFLY ALGORITHM}

Firefly algorithm (FA) was introduced by Yang (2008). FA is inspired by the flashing pattern and characteristics of fireflies where the brightness of a firefly is equal to the objective function value. The lighter firefly (lower fitness value) moves towards brighter firefly (higher fitness value). FA is based on the following idealized behavior of the flashing characteristics of fireflies:

(1) All fireflies are unisex so that one firefly is attracted to other fireflies regardless of their sex.

(2) Attractiveness is proportional to their brightness, thus for any two flashing fireflies, the low brighter one will move towards the high brighter one. The attractiveness is proportional to the brightness and they both decrease as their distance increases. If there is no brighter one than a particular firefly, it will move randomly.

(3) The brightness of a firefly is affected or determined by the landscape of the objective function.

In firefly algorithm, each firefly is assumed as solution to the problem and thereby fitness/brightness (I) is calculated with objective function. In this paper objective function is $H\left(a_{1}, b_{1}, c_{1}, a_{2}, b_{2}, c_{2}\right)$ which is to be maximized by optimizing $a_{1}, b_{1}, c_{1}, a_{2}, b_{2}, c_{2}$ values. So dimensions (D) of the problem are six. Whenever all the firefly fitness values are obtained, firefly whose fitness value is larger among is assigned as brighter firefly. All lighter fireflies (lower fitness value) move towards the brighter firefly by updating their values. Attractiveness $(\beta)$ is varied exponentially with Cartesian distance $\left(r_{i j}\right)$ which is in between brighter firefly $i$ and lighter firefly $\mathrm{j}$. Following is the equation for Cartesian distance between $\mathrm{i}^{\text {th }}$ firefly and $\mathrm{j}^{\text {th }}$ firefly at location $\mathrm{X}_{\mathrm{i}}$ and $\mathrm{X}_{\mathrm{j}}$ respectively.

$$
\text { Cartesian distance }\left(r_{i j}\right)=\left\|X_{i}-X_{j}\right\|=\sqrt{\sum_{k=1}^{N_{c}} \sum_{h=1}^{L}\left(X_{i, k}^{h}-X_{\mathrm{j}, k}^{h}\right)^{2}}
$$

Where $\mathrm{X}_{\mathrm{i}, \mathrm{k}}$ is the $\mathrm{k}^{\text {th }}$ component of the spatial coordinate $\mathrm{X}_{\mathrm{i}}$ of $\mathrm{i}^{\text {th }}$ firefly. Then the attractiveness is given as

$$
\beta=\beta_{0} e^{-\gamma_{i, j}}
$$

Where $\beta$ is the attractiveness at $r_{i, j}=0$ and $\gamma$ is light absorption coefficient of the medium. With this attractiveness, lighter firefly i moves towards brighter firefly $\mathrm{j}$ with the following equation

$$
X_{j, k}^{h}=(1-\beta) X_{\mathrm{i}, k}^{h}+\beta X_{j, k}^{h}+u_{j, k}^{h} \mathrm{k}=1,2, \ldots ., \mathrm{N}, \quad \mathrm{h}=1,2, \ldots \mathrm{D} .
$$

Where $\mathrm{u}$ is a random number that lies between 0 and 1 and is calculated by Eq (23)

$$
u_{i}=\left(\operatorname{rand} 1-\frac{1}{2}\right)
$$

If there is no brighter firefly in the search space then lighter firefly i move randomly with the Eq (24)

$$
X_{\mathrm{i}, k}^{h}=X_{\mathrm{i}, k}^{h}+u_{j, k}^{h} \mathrm{k}=1,2, \ldots . ., \mathrm{N}, \quad \mathrm{h}=1,2, \ldots . \mathrm{D} .
$$

Where rand 1 is a random number lies between 0 and 1

\section{FA-BASED FUZZY ENTROPY METHOD}

In this section, the image thresholding for image segmentation by optimizing/maximizing the fuzzy entropy with proposed ordinary firefly algorithm is explained. The proposed method for image thresholding is very simple and easy to implement. The algorithm of firefly for image thresholding with fuzzy entropy is as follows.

Input: Initialize the population $(\mathrm{N})$, maximum number of iterations, level of thresholding (Th) and its corresponding $a_{1}, b_{1}, c_{1}, a_{2}, b_{2}, c_{2}$ values. Initialize randomization parameter $(\alpha)$, attractiveness $(\beta)$, absorption coefficient $(\gamma)$.

Output: The optimized $a_{1}, b_{1}, c_{1}, a_{2}, b_{2}, c_{2}$ values and its corresponding thresholding values and segmented image.

Initialize all the required parameters and there corresponding dimensions and time $\mathrm{t}=0$.

Calculate the fitness value or light intensity Ii of each solution Xi (i=1,2,3,...n) using Eq. (16) for Fuzzy.

While $(\mathrm{t}<$ Maximum iterations or until termination criteria reached)

for $\mathrm{i}=1: \mathrm{n}$ all $\mathrm{n}$ fireflies 


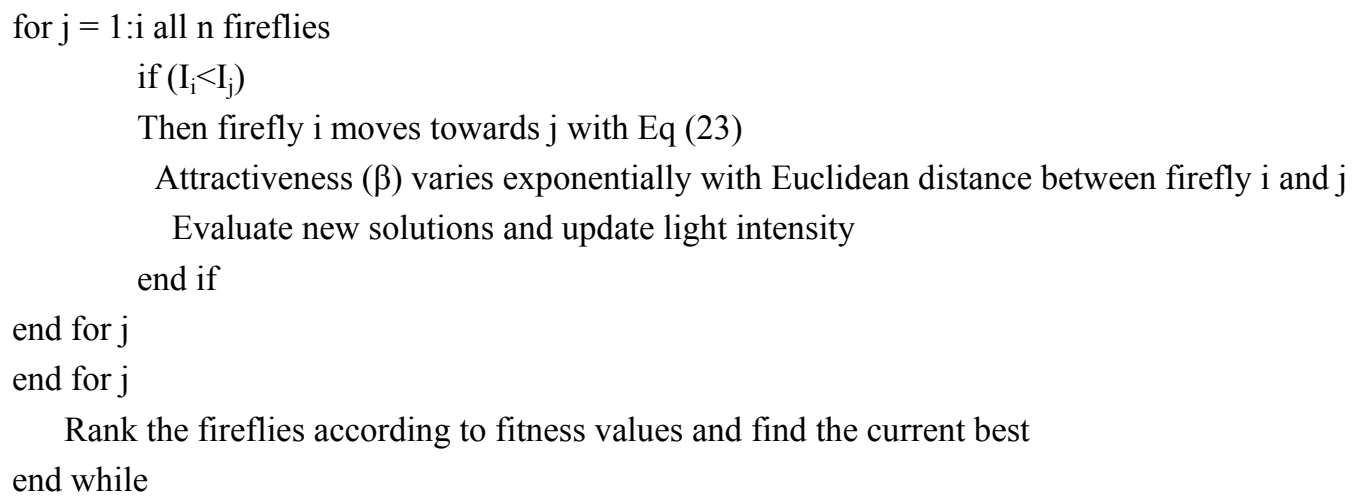

Return the optimal solution and the corresponding segmented image with selected thresholds.

\section{RESULTS AND DISCUSSION}

For the performance evolution which includes robustness, efficiency and convergence of proposed firefly algorithm, researchers selected "Cameraman", "Lena", "Lake", "Goldhill", "Starfish" and "Pirate" as test images. These images are from the image segmentation database whose can be downloaded by anybody through the link (http://www.imageprocessingplace.com/root_files_V3/image_databases.htm) and all are .jpg format images of size $225 \times 225$ and corresponding histograms are shown in Fig. 2. In general, perfect threshold can be selected if the histogram of image peaks is tall, narrow, symmetric, and separated by deep valleys. Cameraman, Lake, Goldhill and pirate image histograms peaks are tall, narrow and symmetric, but for Lena and Starfish images histogram peaks are not tall and narrow so it is difficult to segment with ordinary methods. Hence we proposed a firefly algorithm based image thresholding for effective and efficient image segmentation of above said critical images by optimizing Fuzzy entropy. The performance and effectiveness of proposed firefly algorithm is proved better compared to other optimization techniques like DE, PSO and BA.

\section{A. Selection of DE, PSO, BA and FA parameters}

The same number of populations and maximum number iterations are employed for all optimization algorithms. The maximum number iterations are 30 and population/solutions are 10 times higher of threshold value (i.e if threshold $=2$ then population $=10 \times 2$ ). In DE, Weighting Factor $(\mathrm{F})$ value is 0.5 and Crossover probability (CR) is 0.9 since chosen at these values $\mathrm{DE}$ gives the best results. The performance of PSO algorithm depends on two tuning parameters such as acceleration constants $\left(\mathrm{C}_{1}\right.$ and $\left.\mathrm{C}_{2}\right)$ and inertia weight factor (W). In general $C_{1}$ and $C_{2}$ are set as 2 ; at these values experimentally PSO has given the best fitness values. Whereas inertia weight factor $(\mathrm{W})$ is a random number that lies between 0 and 1.
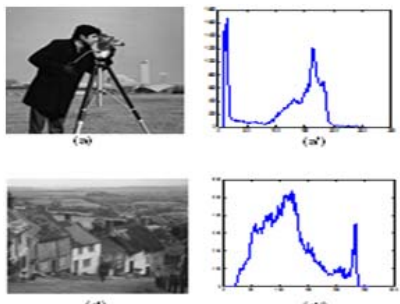
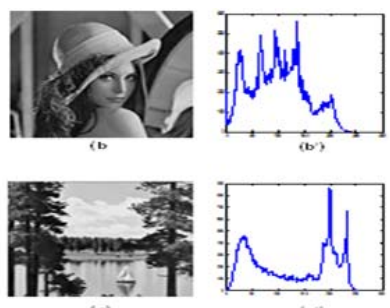
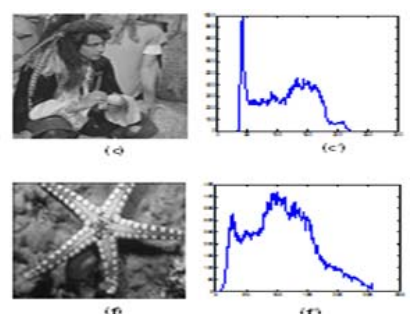

Fig. 2.Test images and corresponding histograms a) Cameraman b) Lena c) Pirate d) Goldhill e) Lake f) Starfish.

The performance of FA depends upon the parameters such as number of solutions (N), dimensions (D), maximum number of iterations (itr), randomization parameter $(\alpha)$, attractiveness $\left(\beta_{0}\right)$ and absorption coefficient $(\gamma)$. While applying FA, these control parameters should be carefully chosen for the successful implementation of the algorithm. Successive experiments were conducted for the selection of these parameters and carry out the best values where objective function is found maximum. Table.Ishows the variation of objective function maximum value, mean and standard deviation with respect to the control parameters for Goldhill image with number of thresholds equal to 2. 50 independent experiments are conducted for fixing FA parameter for validation of the algorithm. From Table.I, it is observed that at $\alpha=0.1, \beta=0.6, \gamma=0.1$ and $N=100$ the objective function value is maximum. These selected parameter values are carried on for all other images. It is observed that beyond $\mathrm{N}=100$, the value of objective function is slightly improved but with the cost of computational time. The parameters of BA such as Loudness $(\mathrm{A}=0.5)$, Pulse rate $(\mathrm{R}=1)$, Frequency minimum $(\mathrm{Qmin}=0)$, Frequency maximum $(\mathrm{Qmax}=30)$, and Step sizes of random walk $(\mathrm{W}=0.001)$ are initialized. 


\begin{tabular}{|c|c|c|c|c|c|c|c|c|c|}
\hline $\begin{array}{c}\text { Parameter } \\
\alpha=0.1\end{array}$ & $\begin{array}{c}\text { Max. } \\
\text { 13.99081 }\end{array}$ & $\begin{array}{l}\text { Mean } \\
13.9831\end{array}$ & $\begin{array}{l}\text { Std.dev. } \\
\mathbf{0 . 0 0 6 0 9}\end{array}$ & Others & $\gamma=0.1$ & 13.9904 & 13.9786 & 0.01629 & \\
\hline$\alpha=0.2$ & 13.986728 & 13.972954 & 0.010783 & & $\gamma=0.2$ & 13.990414 & 13.982511 & 0.005782 & \\
\hline$\alpha=0.3$ & 13.988476 & 13.966132 & 0.019339 & & $\gamma=0.3$ & 13.98982 & 13.98251 & 0.005235 & \\
\hline$\alpha=0.4$ & 13.987238 & 13.952608 & 0.024029 & $\beta=0.4$ & $\gamma=0.4$ & 13.990361 & 13.980398 & 0.011981 & \\
\hline$\alpha=0.5$ & 13.986748 & 13.94969 & 0.026852 & $\gamma=0.5$ & $\gamma=0.5$ & 13.989722 & 13.978113 & 0.011803 & $\alpha=0.1$ \\
\hline$\alpha=0.6$ & 13.988584 & 13.947874 & 0.02408 & $\mathrm{D}=2$ & $\gamma=0.6$ & 13.990156 & 13.984564 & 0.004609 & $\beta=0.6$ \\
\hline$\alpha=0.7$ & 13.986616 & 13.950047 & 0.025424 & $\mathrm{~N}=30$ & $\gamma=0.7$ & 13.988465 & 13.977439 & 0.017647 & $\mathrm{D}=2$ \\
\hline$\alpha=0.8$ & 13.985751 & 13.945179 & 0.027741 & & $\gamma=0.8$ & 13.989284 & 13.980822 & 0.007696 & $\mathrm{~N}=30$ \\
\hline$\alpha=0.9$ & 13.988114 & 13.952208 & 0.020767 & & $\gamma=0.9$ & 13.988399 & 13.975529 & 0.022652 & \\
\hline$\alpha=1$ & 13.983074 & 13.949317 & 0.022222 & & $\gamma=1$ & 13.989032 & 13.978542 & 0.026765 & \\
\hline$\beta=0.1$ & 13.989263 & 13.975598 & 0.014005 & & $\mathrm{p}=20$ & 13.989072 & 13.97491 & 0.022153 & $\alpha=0.1$ \\
\hline$\beta=0.2$ & 13.989782 & 13.980639 & 0.014199 & & $\mathrm{p}=30$ & 13.989734 & 13.975585 & 0.023947 & $\beta=0.6$ \\
\hline$\beta=0.3$ & 13.989642 & 13.981046 & 0.007144 & & $\mathrm{p}=50$ & 13.989899 & 13.981029 & 0.014042 & $\gamma=0.1$ \\
\hline$\beta=0.4$ & 13.990054 & 13.982907 & 0.007186 & & $\mathrm{p}=100$ & 13.99006 & 13.98551 & 0.00373 & $\mathrm{D}=2$ \\
\hline$\beta=0.5$ & 13.989811 & 13.97912 & 0.015729 & $\alpha=0.1$ & & & & & \\
\hline$\beta=0.6$ & 13.99043 & 13.9805 & 0.01483 & $\gamma=0.5$ & & & & & \\
\hline$\beta=0.7$ & 13.990247 & 13.974859 & 0.024922 & $\mathrm{D}=2$ & & & & & \\
\hline$\beta=0.8$ & 13.988599 & 13.97978 & 0.011394 & $\mathrm{~N}=30$ & & & & & \\
\hline$\beta=0.9$ & 13.990187 & 13.981393 & 0.006442 & & & & & & \\
\hline$\beta=1$ & 13.989959 & 13.978749 & 0.012731 & & & & & & \\
\hline
\end{tabular}

To examine the influence of FA algorithm on multilevel thresholding problem, objectives functions/fitness function is Fuzzy entropy

1)Maximization of fuzzy entropy

In this case, the objective function to be optimized with optimization technique is fuzzy entropy which is said to be popular and better in the performance of FA when compared with the other POS, DE and BA. All the algorithms are optimized to maximize the objective function. Table .II and Table.III show the objective values and corresponding thresholds' values for FA, PSO, BA and PSO. It is observed from Table II that the objective value obtained with FA by using fuzzy entropy is higher than DE, PSO and BA for different images.

Table II Comparison of objective values obtained by various algorithms

$\begin{array}{cccccc} & & \text { Th=2 } & \text { Th=3 } & \text { Th }=4 & \text { Th=5 } \\ \text { Images } & \text { Opt Tech } & \text { Fuzzy } & \text { Fuzzy } & \text { Fuzzy } & \text { Fuzzy } \\ \text { Cameraman } & \text { DE } & 13.11179 & 16.51444 & 20.2745 & 23.632996 \\ & \text { PSO } & 13.24719 & 16.54862 & 20.28994 & 23.077552 \\ & \text { BA } & 13.27223 & 16.60881 & 20.41527 & 23.284207 \\ & \text { FA } & 13.34643 & 17.76107 & 21.03949 & 23.524537 \\ \text { Lena } & \text { DE } & 14.12322 & 17.58576 & 20.66932 & 24.0294 \\ & \text { PSO } & 14.22685 & 17.91415 & 21.14748 & 24.300881 \\ & \text { BA } & 14.23849 & 17.93978 & 21.29851 & 24.373777 \\ \text { Goldhill } & \text { FA } & 14.32151 & 17.95987 & 21.38698 & 24.561514 \\ & \text { DE } & 13.44 & 16.82359 & 19.89543 & 22.934799 \\ & \text { PSO } & 13.53301 & 17.0209 & 20.09893 & 22.983463 \\ & \text { BA } & 13.53865 & 17.08749 & 20.16574 & 23.235944 \\ & \text { FA } & 13.56331 & 17.09854 & 20.27302 & 23.360407\end{array}$




\begin{tabular}{cccccc} 
Lake & DE & 13.94999 & 17.32295 & 20.38282 & 23.426922 \\
& PSO & 13.98223 & 17.63517 & 20.87337 & 24.056312 \\
& BA & 13.99141 & 17.71809 & 20.98016 & 24.214624 \\
\multirow{4}{*}{ Pirate } & FA & 14.03941 & 17.69289 & 20.90727 & 24.060597 \\
& DE & 13.87994 & 17.76107 & 21.03949 & 24.373777 \\
& PSO & 14.00623 & 17.91472 & 21.24234 & 24.560984 \\
& BA & 14.01315 & 17.97402 & 21.36288 & 24.766622 \\
Starfish & FA & 14.03124 & 17.69289 & 21.33981 & 24.754253 \\
& DE & 14.46344 & 18.43343 & 21.34769 & 24.375641 \\
& PSO & 14.50729 & 18.25907 & 21.67998 & 24.849605 \\
& BA & 14.51284 & 18.29813 & 21.74343 & 25.104794 \\
& FA & 14.6113 & 18.33766 & 21.79938 & 25.037815
\end{tabular}

\section{Qualitative results}

In this section, researchers concentrated on visual clarity of segmented images with varied threshold values i.e. $\mathrm{Th}=2, \mathrm{Th}=3, \mathrm{Th}=4$ and $\mathrm{Th}=5$ by using Fuzzy entropy with PSO, DE, BA and FA algorithms. The segmented images/thresholding images and corresponding thresholds on histogram obtained with BA and FA algorithms at thresholds level 2, 3, 4 and 5 with Fuzzy entropy are shown in from Fig 3 to Fig 8. Among these figures, we observed that segmented image visual quality is better with higher level of threshold (th $=5$ ) in comparison with $\mathrm{Th}=4, \mathrm{Th}=3$ and $\mathrm{Th}=2$. Let us look on the visual quality of few segmented images with fuzzy entropy for thesake of effectiveness and robustness test of proposed Firefly algorithm. Visual quality of proposed FA is better to BA. (Ex: Lena image at 2 level threshold as shown in Fig. $6 \mathrm{f}$ and Starfish image at 2, 3, 4 and 5- level thresholds as shown in Fig. 8e-h.). Proposed algorithm is better compared to other earlier algorithms in visual quality of image for all other images likewise. The consequence of multilevel thresholding is noticeable from different images. From Fig. 5e, the background in the Lake image is not visibly dissimilar with two level thresholding. But as the number of threshold is extended to 5 (i.e. Fig. $5 \mathrm{~h}$ ), the background becomes recognizable. Similarly in Fig. 8e, the Starfish image mixes up with the background objects. But as the number of threshold is increased to 5 (i.e. Fig. 8h), the Starfish image becomes clearly recognizable.

Table III Optimal threshold values obtained by Fuzzy entropy based evolutionary algorithms.

\begin{tabular}{cccccc} 
Images & Opt Tech & Th=2 & Th=3 & Th=4 & Th=5 \\
Cameraman & DE & 92,220 & $42,134,219$ & $48,94,142,219$ & $44,92,141,191,226$ \\
& PSO & 139,219 & $31,142,192$ & $25,59,132,222$ & $45,94,150,194,231$ \\
& BA & 117,203 & $45,114,184$ & $44,100,159,204$ & $33,81,125,157,208$ \\
& FA & 128,181 & $67,137,202$ & $62,83,152,202$ & $83,129,171,171,173$ \\
Lena & DE & 59,186 & $55,117,189$ & $39,99,140,206$ & $37,80,119,159,207$ \\
& PSO & 71,165 & $68,154,203$ & $69,139,159,200$ & $27,65,101,145,211$ \\
& BA & 68,183 & $68,108,208$ & $59,141,173,215$ & $37,107,147,199,228$ \\
& FA & 55,181 & $78,120,170$ & $36,119,149,193$ & $23,61,110,169,172$ \\
Goldhill & DE & 56,183 & $65,134,196$ & $45,118,153,206$ & $41,87,126,162,209$ \\
& PSO & 53,192 & $60,129,180$ & $32,80,119,200$ & $51,91,118,150,209$ \\
& BA & 113,128 & $47,120,183$ & $44,78,130,208$ & $28,86,113,149,184$ \\
\multirow{4}{*}{ Lake } & FA & 99,167 & $81,141,192$ & $61,129,159,198$ & $47,87,124,161,214$ \\
& DE & 62,189 & $61,124,190$ & $42,109,152,209$ & $38,83,127,169,213$ \\
& PSO & 55,187 & $54,104,178$ & $53,108,152,196$ & $12,64,120,154,201$ \\
& PA & 88,186 & $45,114,184$ & $44,100,159,204$ & $38,88,112,169,203$ \\
& FA & 54,180 & $61,107,189$ & $45,116,130,210$ & $48,81,109,137,214$ \\
& DE & 77,204 & $60,125,192$ & $55,107,138,209$ & $88,130,143,173,213$ \\
& PSO & 74,197 & $61,132,184$ & $63,107,138,182$ & $58,97,129,154,201$
\end{tabular}




\begin{tabular}{lccccc} 
& BA & 80,183 & $53,127,177$ & $51,100,145,199$ & $58,97,129,154,201$ \\
\multirow{3}{*}{ Starfish } & FA & 74,200 & $54,142,179$ & $87,113,162,174$ & $65,119,151,179,216$ \\
& DE & 70,197 & $64,146,210$ & $45,113,159,217$ & $42,85,127,172,217$ \\
& PSO & 57,189 & $39,133,221$ & $50,108,150,211$ & $37,87,115,159,221$ \\
& BA & 128,137 & $128,150,161$ & $75,147,156,197$ & $90,131,132,155,184$ \\
& FA & 76,199 & $44,128,213$ & $56,123,172,218$ & $25,75,108,173,219$
\end{tabular}

\section{Comparison of other methods}

\section{1) Stability analysis}

The optimization technique's outcome is random in nature because randomness is involved in the procedure and the results are not unique for each run. So the algorithm performance is validated by more than one run and with different initial values. An algorithm is said to be robust if its outcome is acceptable (i.e indifferent from one run to another run) under same circumstances. So we run the same algorithm 50 times and considered result at an average of 50 independent runs. The stability of the algorithm is measured with mean and standard deviation. Optimization technique in general can be considered to be better, if its stability factor is higher among all the techniques i.e objective function value should be the same for each run. Mean and standard deviation is calculated by Eq. 25 and Eq. 26

$$
\begin{aligned}
& \operatorname{mean}(\sigma)=\frac{1}{N} \sum_{j}^{N} \mu_{j} \\
& s t d=\sqrt{\frac{1}{N} \sum_{j=1}^{N}\left(\mu_{j}-\sigma\right)^{2}}
\end{aligned}
$$

Where $\mu_{j}$ is the objective function value/fitness value at $\mathrm{j}^{\text {th }}$ run and $\mathrm{N}$ is the number of runs. Table.IV shows the standard deviation values obtained with Fuzzy entropy by proposed firefly algorithm and other algorithms. An

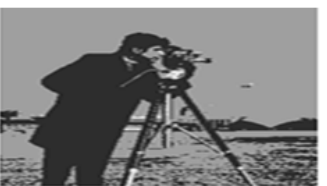

(a)
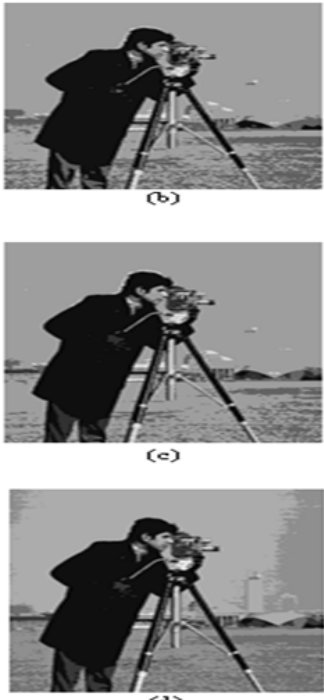

(d)
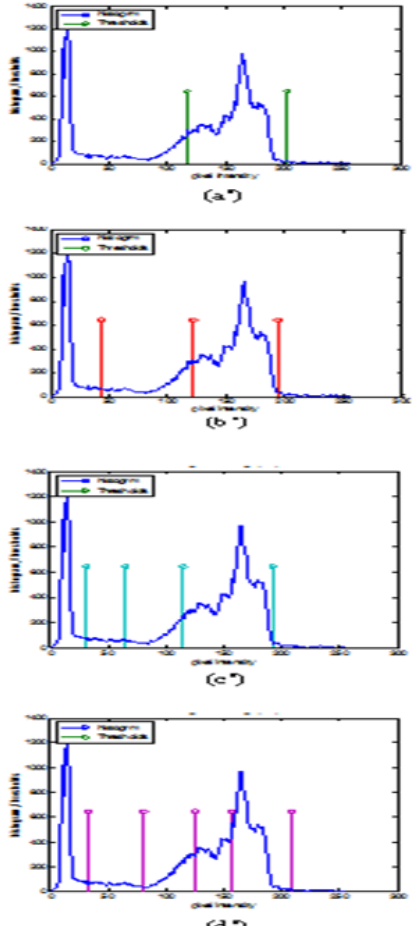

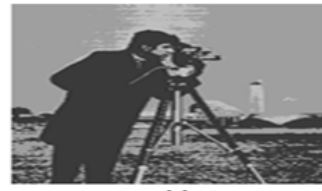

(e)
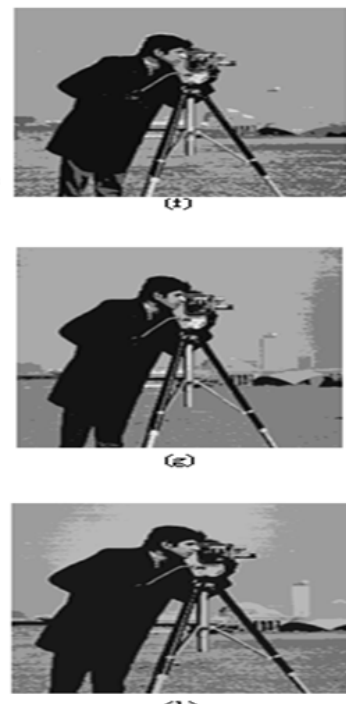

(ro
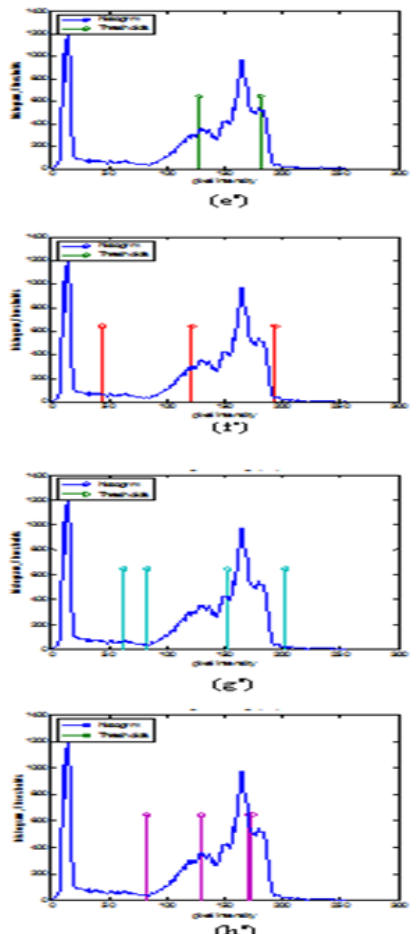

Fig. 3. Segmented images and thresholds on histogram of Cameraman image with various thresholds achieved by BA and FA with Fuzzy entropy. (a)-(d) shows 2-5 level segmented images achieved by BA respectively. (a')-(d') shows 2-5 level thresholds on histogram achieved

by BA respectively. (e)-(h) shows 2-5 level segmented images achieved by FA respectively. (e')-(h') shows 2-5 level thresholds on histogram achieved by FA respectively. 


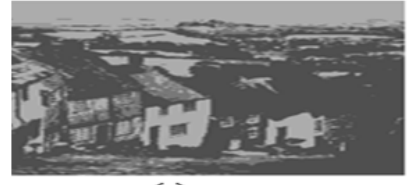

(a)
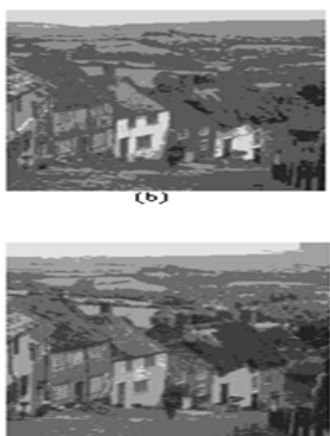

(c)

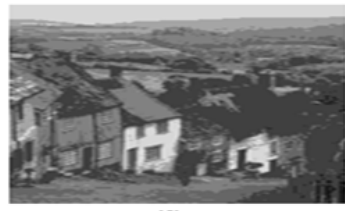

(d)

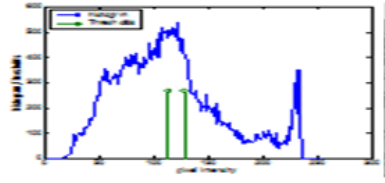

(a)
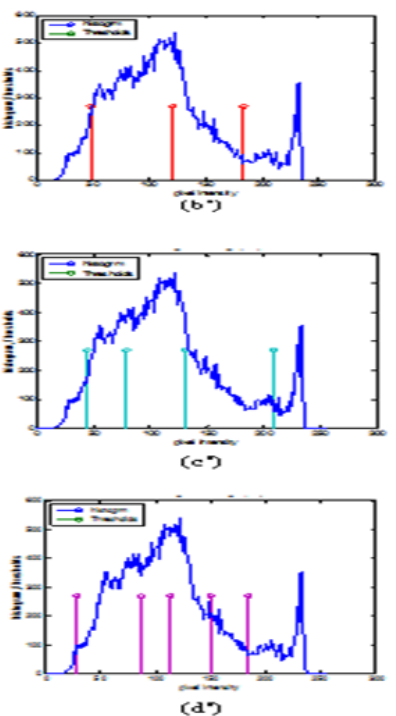

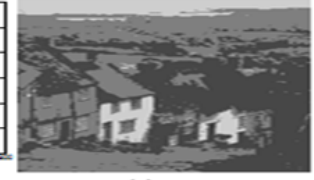

(e)
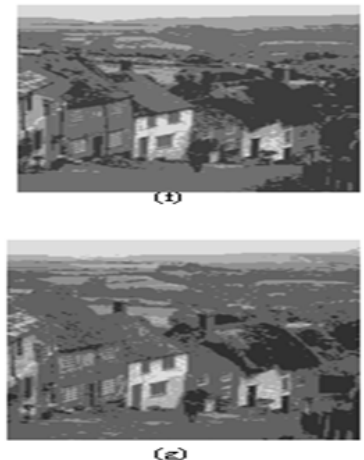

(ख)

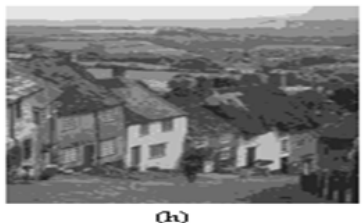

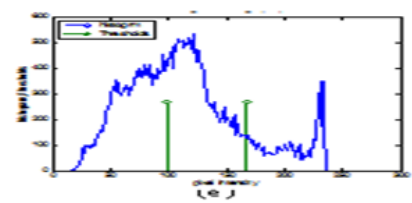
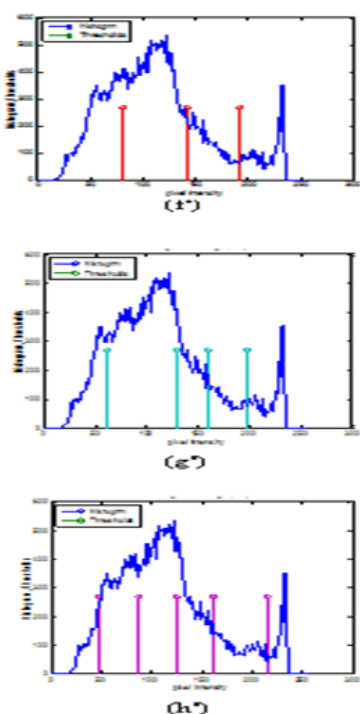

Fig. 4. Segmented images and thresholds on histogram of Goldhill image with various thresholds achieved by BA and FA with Fuzzy entropy. (a)-(d) shows 2-5 level segmented images achieved by BA respectively. (a')-(d') shows 2-5 level thresholds on histogram achieved by BA respectively. (e)-(h) shows 2-5 level segmented images achieved by FA respectively. (e')-(h') shows 2-5 level thresholds on histogram achieved by FA respectively.
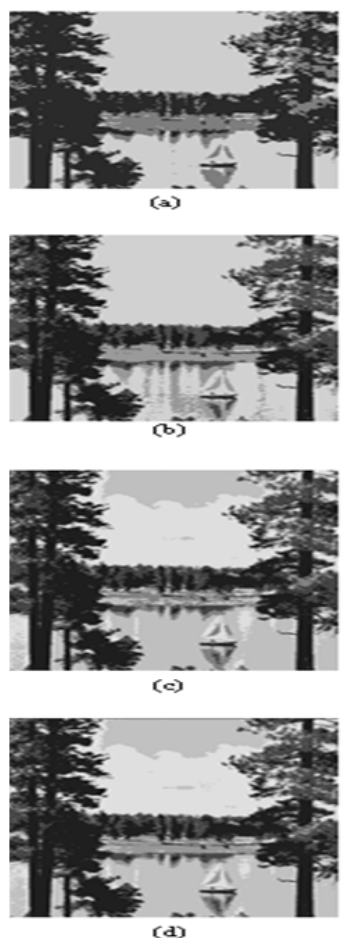

Fig. 5. Segmented images and thresholds on histogram of Lake image with various thresholds achieved by BA and FA with Fuzzy entropy. (a)-(d) shows 2-5 level segmented images achieved by BA respectively. (a')-(d') shows 2-5 level thresholds on histogram achieved by BA respectively. (e)-(h) shows 2-5 level segmented images achieved by FA respectively. (e')-(h') shows 2-5 level thresholds on histogram achieved by FA respectively. 


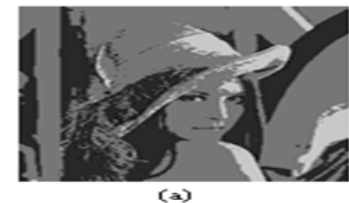

(a)

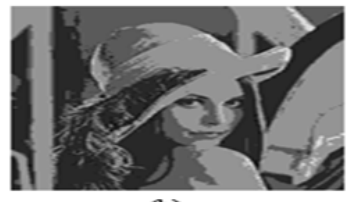

(b)
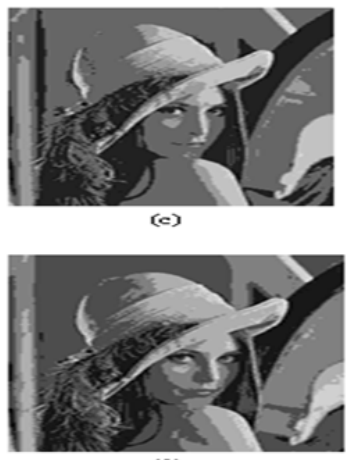

(d)
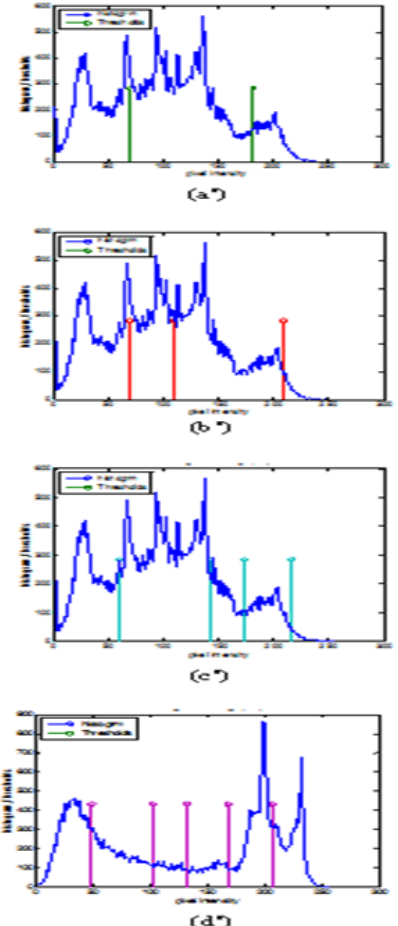

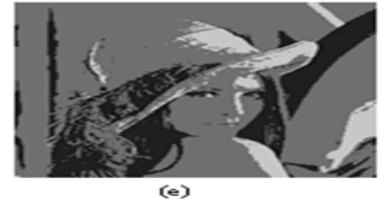

(e)
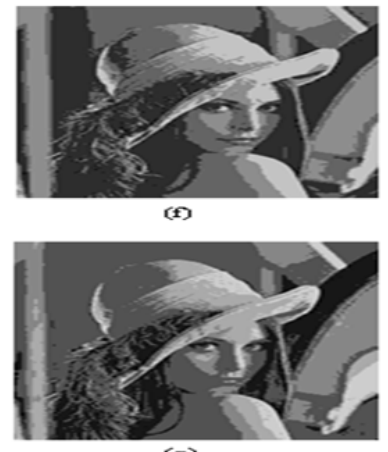

(E)

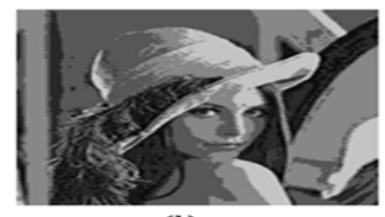

(b)
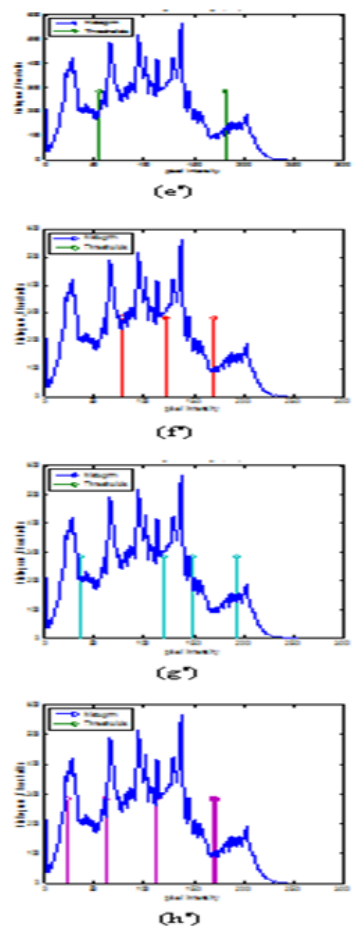

Fig. 6. Segmented images and thresholds on histogram of Lena image with various thresholds achieved by BA and FA with Fuzzy entropy.

(a)-(d) shows 2-5 level segmented images achieved by BA respectively. (a')-(d') shows 2-5 level thresholds on histogram achieved by BA respectively. (e)-(h) shows 2-5 level segmented images achieved by FA respectively. (e')-(h') shows 2-5 level thresholds on histogram achieved by FA respectively.

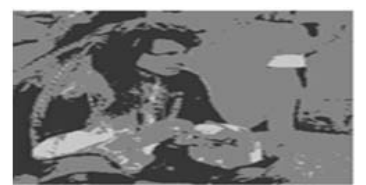

(a)

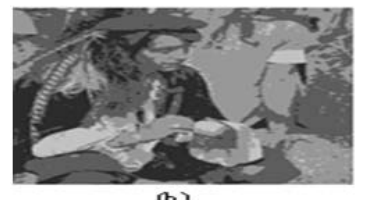

(b)

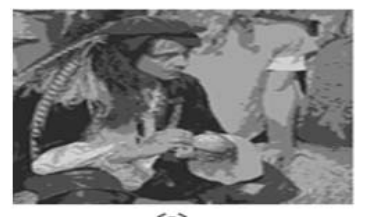

(c)

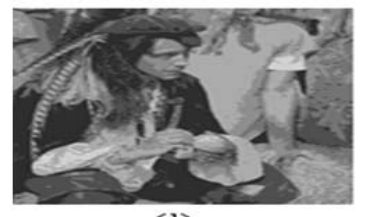

(d)

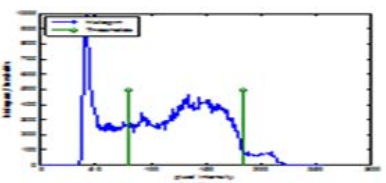

$x=5$
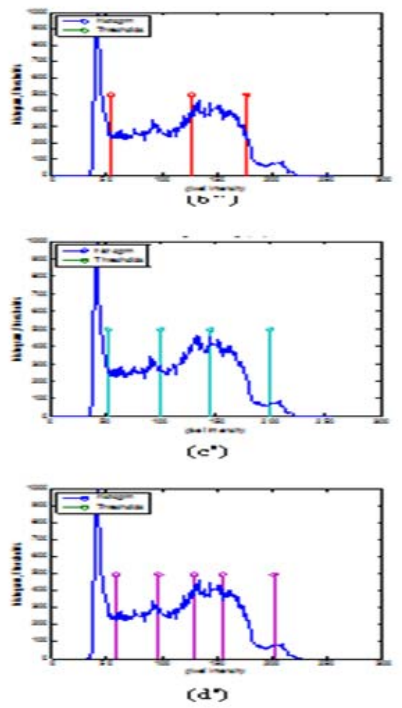

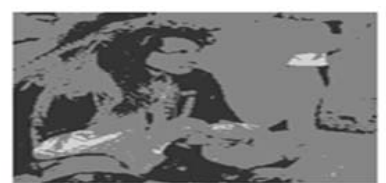

(e)

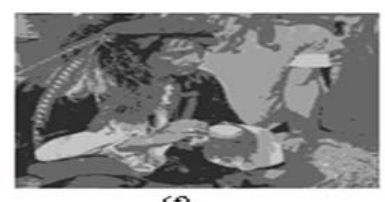

(f)

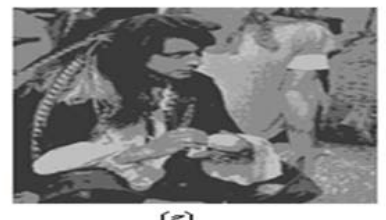

(a)

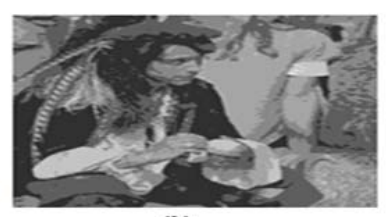

(b)
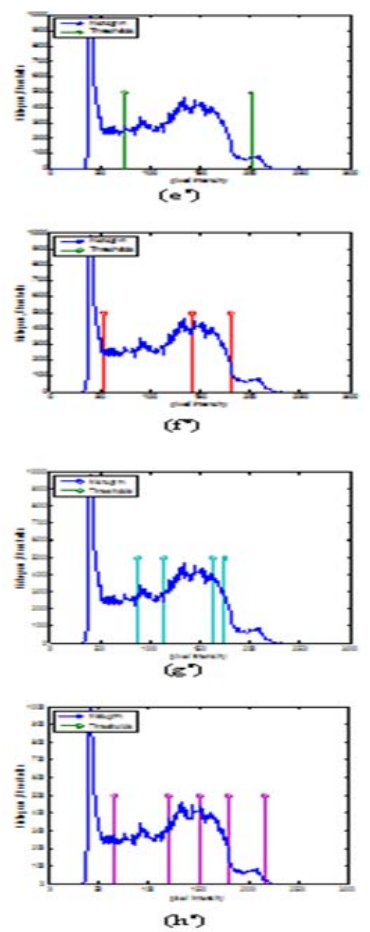

Fig. 7. Segmented images and thresholds on histogram of Pirate image with various thresholds achieved by BA and FA with Fuzzy entropy.

(a)-(d) shows 2-5 level segmented images achieved by BA respectively. (a')-(d') shows 2-5 level thresholds on histogram achieved by BA respectively. (e)-(h) shows 2-5 level segmented images achieved by FA respectively. (e')-(h') shows 2-5 level thresholds on histogram achieved by FA respectively. 


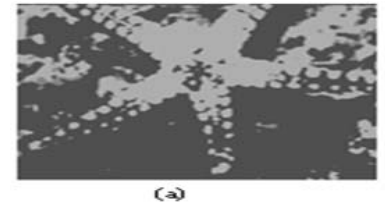

(a)
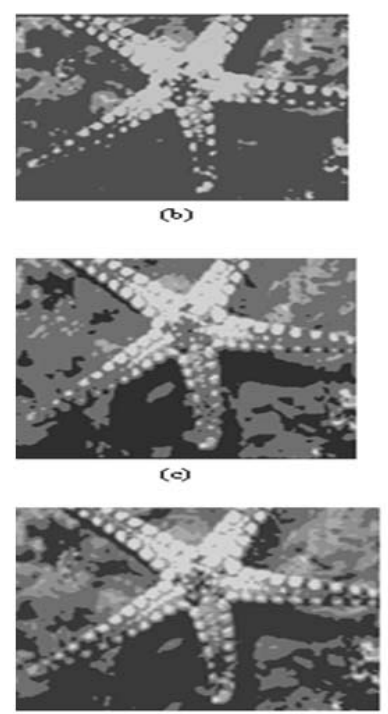

(d)
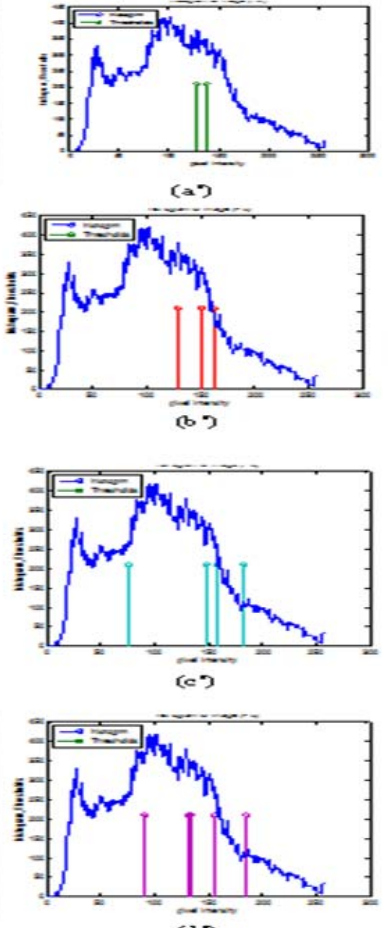

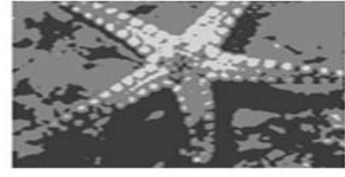

(e)

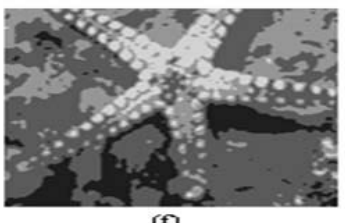

(f)

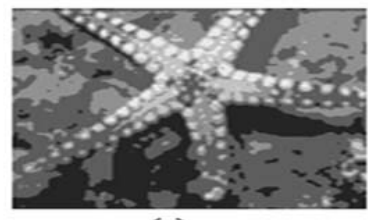

()

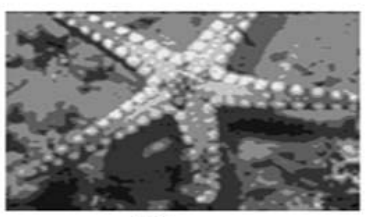

(a)
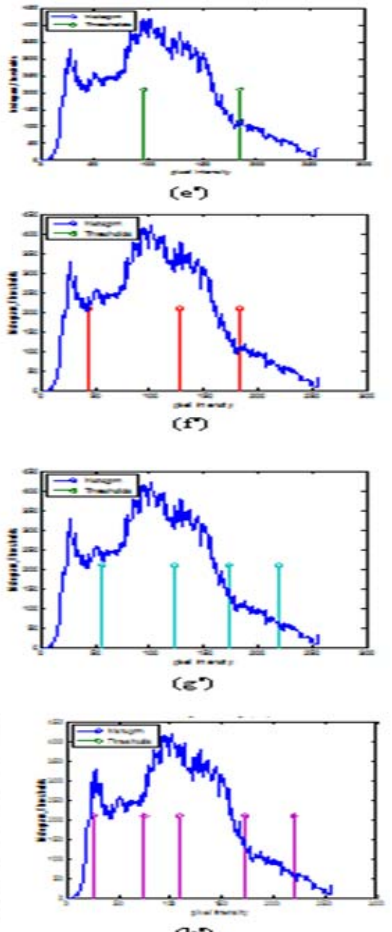

Fig. 8. Segmented images and thresholds on histogram of Starfish image with various thresholds achieved by BA and FA with Fuzzy entropy. (a)-(d) shows 2-5 level segmented images achieved by BA respectively. (a')-(d') shows 2-5 level thresholds on histogram achieved

by BA respectively. (e)-(h) shows 2-5 level segmented images achieved by FA respectively. (e')-(h') shows 2-5 level thresholds on histogram achieved by FA respectively.

optimization technique with higher value of standard deviation seems unstable. From Table.IV, it is observed that DE algorithm has lower standard deviation value for all images including Fuzzy entropy; hence DE is stable and better compared with others. The stability of PSO, BA and FA is found almost similar. It is also observed that there is no effect of standard deviation with the increment of thresholding levels for all images.

Table IV Comparison of standard deviation for various algorithms.

\begin{tabular}{cccccc} 
& & Th=2 & Th=3 & Th $=4$ & Th $=5$ \\
Images & Opt Tech & Fuzzy & Fuzzy & Fuzzy & Fuzzy \\
Cameraman & DE & $1.64 \mathrm{E}-03$ & 0.01668 & $\mathbf{0 . 0 1 7 3 4}$ & 0.00603 \\
& PSO & 0.16129 & $\mathbf{0 . 0 9 2 7 9}$ & 0.17755 & 0.19464 \\
& BA & 0.18212 & $\mathbf{0 . 1 5 9 4 6}$ & 0.21629 & 0.19268 \\
& FA & 0.04025 & 0.04736 & 0.11762 & 0.10343 \\
Lena & DE & $1.56 \mathrm{E}-03$ & $9.82 \mathrm{E}-03$ & 0.01771 & $\mathbf{0 . 0 3 2 7 4}$ \\
& PSO & $\mathbf{0 . 1 2 4 2 2}$ & 0.70027 & 0.19745 & $\mathbf{0 . 1 9 6 1 8}$ \\
& BA & $\mathbf{0 . 1 6 8 2 9}$ & 0.18905 & 0.11919 & 0.23139 \\
\multirow{5}{*}{ Goldhill } & FA & 0.02229 & 0.06475 & 0.07162 & 0.13989 \\
& DE & $8.67 \mathrm{E}-04$ & $1.01 \mathrm{E}-02$ & 0.023675 & 0.038585 \\
& PSO & 0.08252 & 0.173517 & 0.220505 & 0.224984 \\
& BA & 0.167123 & 0.187109 & 0.207755 & $\mathbf{0 . 2 6 1 8 9}$ \\
Lake & FA & 0.023877 & 0.074544 & 0.08749 & 0.148974 \\
& DE & $2.67 \mathrm{E}-04$ & $8.06 \mathrm{E}-01$ & $1.23 \mathrm{E}-02$ & 0.034287 \\
& PSO & $\mathbf{0 . 0 8 0 9 2}$ & 0.002326 & 0.137151 & 0.234356 \\
\multirow{5}{*}{ Pirate } & BA & 0.103968 & 0.159464 & 0.216297 & 0.125343 \\
& FA & 0.012808 & 0.048909 & 0.063312 & 0.109919 \\
& DE & $8.43 \mathrm{E}-04$ & $7.76 \mathrm{E}-03$ & 0.019757 & 0.048521 \\
& PSO & 0.110538 & 0.191169 & 0.203348 & 0.286146
\end{tabular}




\begin{tabular}{lccccc} 
& BA & 0.167082 & 0.291656 & 0.23528 & 0.429339 \\
\multirow{5}{*}{ Starfish } & FA & 0.021899 & 0.056602 & 0.112173 & 0.115502 \\
& DE & $3.16 \mathrm{E}-04$ & $1.20 \mathrm{E}-02$ & $1.93 \mathrm{E}-02$ & 0.029133 \\
& PSO & 0.120118 & 0.164268 & 0.179779 & 0.24098 \\
& BA & $\mathbf{0 . 2 0 3 4 2}$ & $\mathbf{0 . 2 2 3 4 3}$ & 0.210227 & 0.215449 \\
& FA & 0.01713 & 0.043457 & 0.067852 & 0.113278
\end{tabular}

\section{2) Computational complexity:}

It's a measure of time of convergence of an optimization technique which is variable with respect to the thresholds. The computational complexity of Fuzzy entropy is $\mathrm{O}\left(\mathrm{L}^{\mathrm{m}}\right)$ which rises exponentially with the number of thresholds (Th) and the number of gray levels (L). Convergence time of proposed FA depends on the size of image and maximum number of iterations. Table.VIdraw the convergence time/ computational complexities of PSO, DE, BA and FA with Fuzzy entropy for different thresholds for different images. It is observed that average convergence time of proposed FA is much smaller than other algorithms. All the experiments are performed on Matlab 2009b with Intel core i5 processor capacitated 2 GB RAM

3) Peak Signal to Noise Ratio (PSNR):

PSNR shows dissimilarity between threshold image and input image as a measure of visual difference of two images where the units are decibels $(\mathrm{dB})$. A higher value of PSNR indicates better quality of threshold image or reconstructed image. The equation for PSNR is given in Eq. 27.

$$
P S N R=10 \times 10 \log \left(\frac{255^{2}}{M S E}\right)(\mathrm{dB})
$$

Where (MSE) which is given by in Eq. 28

$$
M S E=\frac{1}{M \times N} \sum_{I}^{M} \sum_{J}^{N}\{f(\mathrm{I}, \mathrm{J})-\bar{f}(\mathrm{I}, \mathrm{J})\}^{2}
$$

Where $\mathrm{M} \times \mathrm{N}$ is size of image and I \& $\mathrm{J}$ represent the pixel value of original and decompressed images. In this experiment, researchers have taken $\mathrm{N}=\mathrm{M}$ a square image. $f(I, J)$ is an original image and $\bar{f}(I, J)$ is a reconstructed image of size 225 by 225 . Table. VIshow the PSNR value acquired by different algorithms where the proposed algorithm has achieved higher PSNR value in comparison with DE, PSO and BA. Among all cases as well as in all images, PSNR value is rising with increasing threshold values. FA algorithm provides the utmost value of PSNR value with Th $=5$ when compared to DE, PSO and BA. Hence, the excellence of the segmented images gets better with the higher level of thresholds.

Table V Comparison of CPU time (in seconds) for various methods.

$\begin{array}{cccccc}\text { Images } & & \text { Th=2 } & \text { Th=3 } & \text { Th=4 } & \text { Th=5 } \\ \text { Cameraman } & \text { Opt Tech } & \text { Fuzzy } & \text { Fuzzy } & \text { Fuzzy } & \text { Fuzzy } \\ & \text { DE } & 17.37899 & 34.89404 & 38.9059 & 45.246832 \\ & \text { PSO } & 14.72692 & 22.22853 & 31.88963 & 49.214703 \\ & \text { BA } & 10.92766 & 17.4504 & 28.98167 & 25.063241 \\ & \text { FA } & 23.86838 & 30.19158 & 46.45787 & 81.331957 \\ \text { Lena } & \text { DE } & 23.10234 & 28.87228 & 34.58586 & 60.67167 \\ & \text { PSO } & 20.74006 & 22.81411 & 29.86393 & 45.768399 \\ & \text { BA } & 15.41488 & 16.99354 & 32.38648 & 28.801767 \\ \text { Goldhill } & \text { FA } & 22.1789 & 29.5076 & 44.38185 & 65.414471 \\ & \text { DE } & 27.35428 & 29.93418 & 53.55693 & 54.623062 \\ & \text { PSO } & 20.30875 & 38.3014 & 46.34719 & 66.241249 \\ & \text { BA } & 32.03218 & 20.00902 & 45.73632 & 75.969269 \\ \text { Lake } & \text { FA } & 19.07287 & 42.90798 & 87.7485 & 71.890689 \\ & \text { DE } & 168.5369 & 68.65841 & 42.00675 & 46.557383 \\ & \text { PSO } & 23.40779 & 21.8553 & 37.19785 & 45.344876 \\ & \text { BA } & 22.82106 & 59.24995 & 22.59032 & 40.163425 \\ & \text { FA } & 28.04954 & 29.3333 & 47.70789 & 65.613619\end{array}$




\begin{tabular}{cccccc} 
Pirate & DE & 22.49297 & 27.18165 & 37.74204 & 50.643168 \\
& PSO & 16.9994 & 25.46523 & 36.67485 & 40.139625 \\
& BA & 13.85076 & 14.88508 & 25.45828 & 87.11891 \\
\multirow{4}{*}{ Starfish } & FA & 19.67271 & 37.68114 & 48.68866 & 70.158385 \\
& DE & 26.33895 & 26.22158 & 62.34102 & 56.571056 \\
& PSO & 26.68756 & 24.80893 & 33.89914 & 42.17283 \\
& BA & 13.06687 & 40.29626 & 22.25709 & 27.008018 \\
& FA & 22.89818 & 29.1351 & 58.27002 & 66.192443
\end{tabular}

4) Misclassification error/Uniformity measure:

It is measure of uniformity in threshold image and is used to compare optimization techniques performance (Sahoo et al., 1988). Misclassification error is measured by Eq. 29

$$
M=1-2 * T h * \frac{\sum_{j=0}^{T h} \sum_{i \in R_{j}}\left(I_{i}-\sigma_{j}\right)^{2}}{N *\left(I_{\max }-I_{\min }\right)^{2}}
$$

Where $T$ is the number of thresholds that are used to segment the image, $R_{j}$ is the jth segmented region, $I_{i}$ is the intensity level of pixel in that particular segmented area, $\sigma_{\mathrm{j}}$ is the mean of $\mathrm{j}^{\text {th }}$ segmented region of image, $\mathrm{N}$ is total number of pixels in the image and $I_{\min } \& I_{\max }$ are the maximum and minimum intensity of image respectively. In general misclassification errors lie between $0 \& 1$ and higher value of misclassification error shows better performance of the algorithm. Hence, the Uniformity measure in thresholding is measured from the difference between maximum value, 1 (better quality of image) and minimum value, 0 (worst quality of image). Table.VII demonstrate misclassification error of proposed and other techniques where the proven proposed method has lesser misclassification error and draws better visual quality.

Table VI Comparison of PSNR values for the methods under evaluation.

$\begin{array}{cccccc} & & \text { Th=2 } & \text { Th=3 } & \text { Th=4 } & \text { Th=5 } \\ \text { Images } & \text { Opt Tech } & \text { Fuzzy } & \text { Fuzzy } & \text { Fuzzy } & \text { Fuzzy } \\ \text { Cameraman } & \text { DE } & 28.677 & 29.5452 & 30.002 & 30.991 \\ & \text { PSO } & 28.7008 & 29.9306 & 30.5631 & 31.1215 \\ & \text { BA } & 28.9523 & 30.0574 & 30.6835 & 31.5316 \\ & \text { FA } & 29.0196 & 30.3965 & 31.0511 & 32.0426 \\ \text { Lena } & \text { DE } & 28.6276 & 28.6481 & 29.1021 & 29.8851 \\ & \text { PSO } & 28.7274 & 29.3717 & 29.1572 & 30.1187 \\ & \text { BA } & 28.742 & 29.5076 & 29.5213 & 30.2771 \\ & \text { FA } & 28.8438 & 29.6273 & 29.745 & 30.9163 \\ \text { Goldhill } & \text { DE } & 28.8427 & 28.9637 & 29.2554 & 30.6413 \\ & \text { PSO } & 29.0318 & 29.1168 & 29.5548 & 30.7302 \\ & \text { BA } & 29.0657 & 29.4898 & 30.0733 & 30.9964 \\ \text { Lake } & \text { FA } & 29.1103 & 29.5282 & 30.2903 & 31.481 \\ & \text { DE } & 28.8852 & 29.3218 & 29.9428 & 30.6342 \\ & \text { PSO } & 28.9172 & 29.6214 & 30.0652 & 30.8547 \\ & \text { BA } & 29.0525 & 29.7387 & 30.6819 & 31.5673 \\ \text { Pirate } & \text { FA } & 29.3006 & 33.3683 & 31.1231 & 31.7122 \\ & \text { DE } & 28.7004 & 29.5453 & 30.3487 & 30.5687 \\ & \text { PSO } & 28.7038 & 29.7589 & 30.8305 & 31.0817 \\ & \text { BA } & 28.7385 & 29.8286 & 31.1737 & 31.2118 \\ & \text { FA } & 28.7525 & 29.8614 & 31.2954 & 32.2861 \\ & \text { DE } & 28.7096 & 28.6613 & 29.306 & 29.7045 \\ & \text { PSO } & 28.32849 & 28.9917 & 29.4915 & 30.1118 \\ & \text { BA } & 28.7013 & 29.0443 & 29.5343 & 30.2872 \\ & \text { FA } & 28.70678 & 29.2359 & 29.5676 & 30.5047\end{array}$


5) Structural Similarity Index (SSIM):

It evaluates the visual similarity between the original image and the reconstructed image/thresholded image and is calculated with below equation

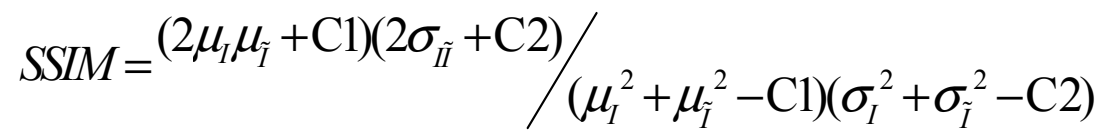

Where $\mu \mathrm{I}$ and $\mu \tilde{I}$ are the mean value of the original image I and reconstructed image $\tilde{\mathrm{I}}, \sigma_{\mathrm{I}}$ and $\sigma_{\mathrm{I}}$ are the standard deviation of original image I and reconstructed image $\tilde{\mathrm{I}}, \sigma_{\text {IĨ }}$ is the cross-correlation and C1 \& $\mathrm{C} 2$ are constants which are equal to 0.065 . The range of SSIM is -1 to +1 and SSIM value equal to one shows original image and reconstructed image/thresolded image is similar. Algorithm is said to be good if SSIM value is near around positive one. Table.VIII show the SSIM of various methods with Fuzzy entropy and it shows that the proposed method SSIM is higher than other methods.

$$
\text { Standard deviation } \sigma_{\tilde{I I}}=\frac{1}{N-1} \sum_{i=1}^{N}\left(\mathrm{I}_{i}-\mu_{I}\right)\left(\tilde{\mathrm{I}}_{i}-\mu_{\tilde{I}}\right)
$$

Table VII Comparative misclassification error for various thresholding methods.

$\begin{array}{cccccc} & & \text { Th=2 } & \text { Th=3 } & \text { Th }=4 & \text { Th=5 } \\ \text { Images } & \text { Opt Tech } & \text { Fuzzy } & \text { Fuzzy } & \text { Fuzzy } & \text { Fuzzy } \\ \text { Cameraman } & \text { DE } & 0.965365 & 0.977768 & 0.927555 & 0.9075964 \\ & \text { PSO } & 0.951125 & 0.975786 & 0.924084 & 0.9449547 \\ & \text { BA } & 0.936811 & 0.972633 & 0.899591 & 0.9138578 \\ & \text { FA } & 0.916367 & 0.936172 & 0.882454 & 0.878416 \\ \text { Lena } & \text { DE } & 0.968633 & 0.95537 & 0.939388 & 0.8932978 \\ & \text { PSO } & 0.959245 & 0.952794 & 0.935731 & 0.8073844 \\ & \text { BA } & 0.951017 & 0.949908 & 0.917558 & 0.7287992 \\ \text { Goldhill } & \text { FA } & 0.948264 & 0.946852 & 0.902922 & 0.7233069 \\ & \text { DE } & 0.971564 & 0.950803 & 0.895224 & 0.6886802 \\ & \text { PSO } & 0.947282 & 0.938885 & 0.853442 & 0.6833011 \\ & \text { BA } & 0.938959 & 0.92963 & 0.748672 & 0.6637794 \\ \text { Lake } & \text { FA } & 0.930716 & 0.909869 & 0.723765 & 0.6179214 \\ & \text { DE } & 1.369244 & 0.948094 & 0.892369 & 0.7512455 \\ & \text { PSO } & 0.96305 & 0.971748 & 0.889823 & 0.6881701 \\ & \text { BA } & 0.954991 & 0.936172 & 0.88658 & 0.6263704 \\ \text { Pirate } & \text { FA } & 0.953434 & 0.931674 & 0.882454 & 0.5644232 \\ & \text { DE } & 0.946298 & 0.951736 & 0.918854 & 0.9060872 \\ & \text { PSO } & 0.933165 & 0.95061 & 0.903981 & 0.905192 \\ & \text { BA } & 0.931228 & 0.944304 & 0.900143 & 0.871334 \\ \text { Starfish } & \text { FA } & 0.930858 & 0.933864 & 0.896563 & 0.8350455 \\ & \text { DE } & 0.95527 & 0.974187 & 0.928185 & 0.8560859 \\ & \text { PSO } & 0.952507 & 0.953383 & 0.927381 & 0.7894524 \\ & \text { BA } & 0.94859 & 0.939 & 0.910614 & 0.7713202 \\ & \text { FA } & 0.943572 & 0.915473 & 0.902217 & 0.7304659\end{array}$


Table VIII Comparison of structural similarity index (SSIM) for various algorithms.

\begin{tabular}{|c|c|c|c|c|c|}
\hline & & $\mathrm{Th}=2$ & $\mathrm{Th}=3$ & $\mathrm{Th}=4$ & $\mathrm{Th}=5$ \\
\hline Images & Opt Tech & Fuzzy & Fuzzy & Fuzzy & Fuzzy \\
\hline \multirow[t]{4}{*}{ Cameraman } & $\mathrm{DE}$ & 0.637394 & 0.799621 & 0.784806 & 0.8200447 \\
\hline & PSO & 0.649298 & 0.816236 & 0.843798 & 0.8252173 \\
\hline & BA & 0.698512 & 0.816741 & 0.847386 & 0.8736756 \\
\hline & FA & 0.745811 & 0.820292 & 0.84779 & 0.8562776 \\
\hline \multirow[t]{4}{*}{ Lena } & $\mathrm{DE}$ & 0.656616 & 0.747751 & 0.770816 & 0.8210519 \\
\hline & PSO & 0.663094 & 0.711089 & 0.734453 & 0.7670472 \\
\hline & BA & 0.663156 & 0.745134 & 0.745062 & 0.7895847 \\
\hline & FA & 0.695085 & 0.700278 & 0.732765 & 0.7979711 \\
\hline \multirow[t]{4}{*}{ Goldhill } & $\mathrm{DE}$ & 0.441218 & 0.644457 & 0.670154 & 0.7810997 \\
\hline & PSO & 0.474546 & 0.667122 & 0.689939 & 0.7909164 \\
\hline & $\mathrm{BA}$ & 0.606807 & 0.668688 & 0.727309 & 0.7915057 \\
\hline & FA & 0.645513 & 0.694545 & 0.732217 & 0.8096297 \\
\hline \multirow[t]{4}{*}{ Lake } & $\mathrm{DE}$ & 0.688739 & 0.805535 & 0.835122 & 0.8434764 \\
\hline & PSO & 0.672471 & 0.987456 & 0.824017 & 0.8463463 \\
\hline & BA & 0.719987 & 0.799621 & 0.847386 & 0.8756467 \\
\hline & FA & 0.710761 & 0.795692 & 0.816913 & 0.8773202 \\
\hline \multirow[t]{4}{*}{ Pirate } & $\mathrm{DE}$ & 0.562101 & 0.662256 & 0.763526 & 0.7811629 \\
\hline & PSO & 0.565558 & 0.704156 & 0.784691 & 0.8029126 \\
\hline & BA & 0.566939 & 0.716693 & 0.785478 & 0.8158027 \\
\hline & FA & 0.611312 & 0.720464 & 0.819659 & 0.8431184 \\
\hline \multirow[t]{4}{*}{ Starfish } & $\mathrm{DE}$ & 0.493903 & 0.541492 & 0.653287 & 0.7067376 \\
\hline & PSO & 0.494132 & 0.565434 & 0.691855 & 0.7283583 \\
\hline & BA & 0.517186 & 0.620772 & 0.696124 & 0.7667584 \\
\hline & FA & 0.525031 & 0.765436 & 0.719282 & 0.7724659 \\
\hline
\end{tabular}

\section{CONCLUSIONS}

A firefly algorithm based multilevel image thresholding for image segmentation has been productively proposed with desired output. Firefly algorithm maximizes the Fuzzy entropy for the efficient and effective image thresholding. The proposed algorithm is tested on natural images to show the merits of algorithm. Results of the proposed method are compared with other optimization techniques such as DE, PSO and BA with Fuzzy entropy. It is observed that proposed algorithm has higher/maximum fitness value compared to DE, PSO and BA. The PSNR value shows higher values with proposed algorithm than DE, PSO and BA and thereby draws better quality of the segmented image with proposed method. It can be concluded that proposed algorithm outperform the DE, PSO and BA in all performance measuring parameters. Further in future scope the firefly algorithm convergence time and efficiency is improved by modifying the algorithm process.

\section{Reference:}

[1] Agrawal. S, R. Panda, S. Bhuyan, B.K. Panigrahi, "Tsallis entropy based optimal multilevel thresholding using cuckoo search algorithm", Swarm and Evolutionary Computation, Vol. 11 pp. 16-30, 2013.

[2] Akay. B, "A study on particle swarm optimization and artificial bee colony algorithms for multilevel thresholding", Applied Soft Computing Vol. 13, pp. 3066-3091, 2013.

[3] Ayala. H, F. Santos, V. Mariani and L. Coelho, "Image thresholding segmentation based on a novel beta differential evolution approach", Expert Systems with Applications, Vol. 42, pp. 2136-2142, 2015.

[4] Bhandari. A. K, A. Kumar, G. K. Singh, "Tsallis entropy based multilevel thresholding for colored satellite image segmentation using evolutionary algorithms", Expert Systems With Applications, Vol. 42, pp. 8707-8730, 2015.

[5] Bhandari. A. K, A. Kumar, G. K. Singh, " Modified artificial bee colony based computationally efficient multilevel thresholding for satellite image segmentation using Kapur's, Otsu and Tsallis functions", Expert Systems With Applications, Vol. 42, pp. 1573-1601, 2015.

[6] Chiranjeevi, K. and Umaranjan. J. (2016) 'Fast vector quantization using a Bat algorithm for image compression', Engineering Science and Technology, an International Journal, Vol. 19, pp. 769-781.

[7] De Luca. A, S. Termini, A definition of a non-probabilistic entropy in the setting of fuzzy sets theory, Inf. Control 20 (1972) 301-312. 
[8] Horng. M and T. Jiang, "Multilevel Image Thresholding Selection based on the Firefly Algorithm", Symposia and Workshops on Ubiquitous, Autonomic and Trusted Computing, pp. 58-63, 2010.

[9] Hussein. W. A, S. Sahran , S. Abdullah, "A fast scheme for multilevel thresholding base d on a modifie d b ees algorithm", Knowledge-Based Systems, Vol. 101, pp. 114-134, 2016.

[10] Kapur. J. N, P.K.Sahoo, A.K.C Wong, A new method for gray-level picture thresholding using the entropy of the histogram”, Computer Vision Graphics Image Process. 29 (1985) $273-285$.

[11] Li. Y, L. Jiao, R. Shang, R. Stolkin, "Dynamic-context cooperative quantum-behaved particle swarm optimization based on multilevel thresholding applied to medical image segmentation”, Information Sciences, Vol. 294, pp. 408-422, 2015.

[12] Mbuyamba. M, J. Cruz-Duarte , J. Avina-Cervantes, C. Correa-Cely, D. Lindner, and C. Chalopin, "Active contours driven by Cuckoo Search strategy for brain tumour images segmentation”, Expert Systems With Applications, Vol. 56, pp. 59-68, 2016.

[13] Otsu. N, "A threshold selection from gray level histograms" IEEE Transactions on System, Man and Cybernetics 66, 1979.

[14] Ouadfel. S and A. Taleb-Ahmed, "Social spiders optimization and flower pollination algorithm for multilevel image thresholding: A performance study", Expert Systems with Applications, Vol. 55 pp. 566-584, 2016.

[15] Penga. H, J. Wangb, M. Pérez-Jiménezc, "Optimal multi-level thresholding with membrane computing", Digital Signal Processing, Vol. 37, pp. 53-64, 2015.

[16] Saha. S. .I, Siddhartha Bhattacharyy and Ujjwal Maulik, "Multi-level thresholding using quantum inspired meta-heuristics", Knowledge-Based Systems 67 (2014) 373-400.

[17] Sahoo, P. K., Soltani, S., Wong, A. K. C., \& Chen, Y. C. (1988). A survey of thresholding techniques. Computer Vision Graphics Image Processing, 41, 233-260.

[18] Sathya. P. D and R. Kayalvizhi, "Optimal multilevel thresholding using bacterial foraging algorithm", Expert Systems with Applications, Vol. 38, pp. 15549-15564, 2011.

[19] Sathya. P. D and R. Kayalvizhi, "Modified bacterial foraging algorithm based multilevel thresholding for image segmentation", Engineering Applications of Artificial Intelligence, Vol. 24, pp. 595-615, 2011.

[20] Sathya. P. D and R. Kayalvizhi, "Amended bacterial foraging algorithm for multilevel thresholding of magnetic resonance brain images", Measurement, Vol. 44, pp. 1828-1848, 2011.

[21] Sathya. P. D and R. Kayalvizhi, "Optimal segmentation of brain MRI based on adaptive bacterial foraging algorithm", Neurocomputing, Vol. 74, pp. 2299-2313, 2011.

[22] Sezgin. M, B. Sankur, Survey over image thresholding techniques and quantitative performance evaluation, J. Electron. Imaging 13 (1) (2004) 146-165.

[23] Sun. G, A. Zhang, Y. Yao and Z. Wang, "A novel hybrid algorithm of gravitational search algorithm withgenetic algorithm for multilevel thresholding", Applied Soft Computing, 2016.

[24] Tao. W, H. Jin, L. Liu, Object segmentation using ant colony optimization algorithm and fuzzy entropy, Pattern Recognit. Lett. 28 (2007) 788-796.

[25] Yang. X. S," Nature-Inspired Metaheuristic Algorithms", Luniver Press, 2008.

[26] Ye. Z, M. Wang, W. Liu, S. Chen, "Fuzzy entropy based optimal thresholding using bat algorithm”, Applied Soft Computing, Vol. 31, pp. 381-395, 2015.

[27] Zhao. M, A.M. Fu, H. Yan, A technique of three-level thresholding based on probability partition and fuzzy 3-partition, IEEE Trans. Fuzzy Syst. 9 (2001) 469-479. 